\title{
Correction to: Limb Remote Ischemic Preconditioning Reduces Repeated Ketamine Exposure-Induced Adverse Effects in the Developing Brain of Rats
}

\author{
Ying Liu ${ }^{1} \cdot$ An Qi $\mathrm{Li}^{1} \cdot$ Wan $\mathrm{Ma}^{2} \cdot \mathrm{Yu}$ Bo Gao ${ }^{2} \cdot \mathrm{Li}$ Qin Deng ${ }^{2} \cdot$ Chun Zhang ${ }^{3} \cdot$ Jin Hai Meng $^{2}$ (D)
}

Published online: 24 February 2020

(C) Springer Science+Business Media, LLC, part of Springer Nature 2020

Correction to: Journal of Molecular Neuroscience (2019) 68:58-65

https://doi.org/10.1007/s12031-019-01282-3

The original version of this article unfortunately contained mistakes in Fig. 2c, d and in the "Funding Information" section:

1. The protein internal reference "GAPDH" was incorrectly captured as "GADPH" in Fig. 2c, d. The updated Figure is presented below:
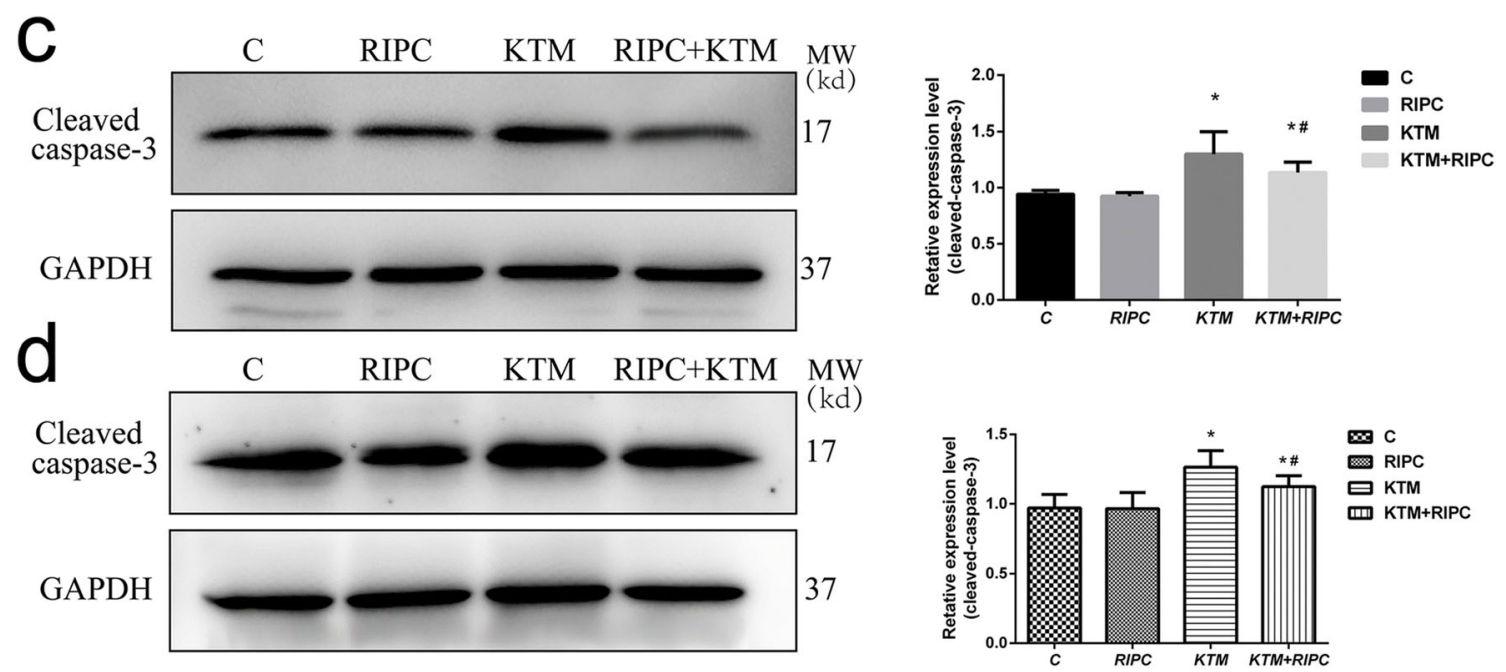

The online version of the original article can be found at https://doi.org/10. 1007/s12031-019-01282-3

Jin Hai Meng

mengjinhai2616@163.com

1 Department of Anaesthesiology, Ningxia Medical University, Yinchuan, Ningxia, China
Department of Anaesthesiology, General Hospital of Ningxia Medical University, Shengli Street, Yinchuan 750004, Ningxia, China

3 Department of Key Laboratory of Cerebral Diseases, Ningxia Medical University, Yinchuan, Ningxia, China 
2. The designated grant number 81560225 of the National Natural Science Foundation of China was incorrectly captured as 81560502 . The updated funding statement is presented below:

Funding Information This work was supported by grants from the National Natural Science Foundation of China (81560502) and the Ningxia Natural Science Foundation (NZ15143 to Jinhai Meng and NZ16147 to Wan MA).
Publisher's Note Springer Nature remains neutral with regard to jurisdictional claims in published maps and institutional affiliations. 